\title{
Ameliorative effects of curcumin on microvascular complication streptozotocin-induced diabetic rat penis
}

\section{Wipapan Khimmaktong1, Manaras Komolkriengkrai ${ }^{1}$, Piyakorn Boonyoung ${ }^{1}$ and Vipavee Anupunpisit ${ }^{2}$}

\begin{abstract}
${ }^{1}$ Department of Anatomy, Faculty of Science, Prince of Songkla University, Songkhla, Thailand, ${ }^{2}$ Department of Anatomy, Faculty of Medicine, Srinakharinwirot University, Bangkok, Thailand
\end{abstract}

Corresponding Author: Wipapan Khimmaktong PhD, Department of Anatomy, Faculty of Science, Prince of Songkla University, Hatyai, Songkhla 90110, Thailand.

Submission Date: October $3^{\text {rd }}$, 2018, Acceptance Date: February $25^{\text {th }}$, 2019, Publication Date: February $28^{\text {th }}, 2019$

Citation: Khimmaktong W., Komolkriengkrai M., Boonyoung P., Anupunpisit V.. Ameliorative effects of curcumin on microvascular complicationin streptozotocin-induced diabetic rat penis. Functional Foods in Health and Disease 2019; 9(2): 92-107. DOI: https://doi.org/10.31989/ffhd.v9i2.553

\begin{abstract}
Background: Vascular diseases from diabetes typically result in conditions that interfere with the microvascular functions, such as erectile dysfunction (ED) of the penis. It is unrevealed which morphological changes the microvasculature of the penis may result from the treatment and supplementation of curcumin.
\end{abstract}

Objective: This study aims to determine the effects of curcumin, the active compound of turmeric, on penile microvasculature in experimentally induced diabetic rats.

Methods: Rat were divided into three groups, control (C) and diabetic (DM). Diabetic rats supplemented with curcumin (DMC) $(200 \mathrm{mg} / \mathrm{kg} \mathrm{BW})$. For induction of a diabetic condition, rats were injected with a single intraperitoneal injection of streptozotocin $(60 \mathrm{mg} / \mathrm{kg} \mathrm{BW})$. A histological study and vascular corrosion casting along with scanning electron microscopy (SEM) were used to determine a morphological change of microvessels in the penis of rats after 8 weeks of experiments.

Results: The histological investigations of penile tissue of DM group revealed thickly lined venous sinuses with smooth but sparse muscle layers. Dorsal arteries (DA) and deep dorsal veins (DV) of the penis were present of narrow lumen and showed thickening of collagen fibers at their wall of tunica media. An increasing thickness of collagen fibers was observed. Vascular corrosion casting revealed critically decreased diameters of DM venous sinus in the penis' glans, which were observed as shrunken and partially lacking in the distal area of the glans. 
The diameter of venous sinuses in corpus cavernosum (CC) of DM rats were dilated and the density of venous sinuses was decreased. Interestingly, the signs of vessel restoration and improvement were presented by increasing diameters and recovering with healthy and nourished DA, DV, venous sinus at the area of the glans, and CC of the penis in DMC groups.

Conclusion: The results have shown that the effects of curcumin could improve the destruction of the penile microvasculature in induced diabetic rats by STZ.

Keywords: Diabetes mellitus, streptozotocin, curcumin, penis, Microvasculature, vascular corrosion cast

\section{BACKGROUND}

Abnormal blood vessels which become narrow and harden cause problems in many diseases. High blood sugar levels or hyperglycemia in diabetes mellitus is the most common cause of blood vessel alteration. The vascular disease is a typical condition interfering with the ability to maintain an erection, or erectile dysfunction (ED), that prevents the blood flow into and out of the penis. ED is widely regarded as a result of the prevalence of obesity and metabolic syndrome. About $50 \%$ of diabetic men experience pain from ED [1]. Diabetes is not only a risk factor for $\mathrm{ED}$, but also one of the most difficult risk factors to treat [2]. The disease progression likely brings down the effectiveness of standard alternatives for the treatment of ED in diabetic men. Finally, penile prosthesis surgery is the end-stage treatment for a large group of patients. A common cause of ED was penile venous insufficiency or venogenic ED, also called a venous leak.

In long-term hyperglycemia, the vascular tissue does not completely react to nitric oxide and the endogenous production of nitric oxide is not abundant. The endothelium is a key switch of the production of nitric oxide (NO) from vascular smooth muscles. Misplacement of endothelial cells in diabetes-induced rats leads to impairment of vascular function [3-4]. When the body has sufficient blood flow into the penis, penile veins confine and close off the blood from flowing out. This action leads to an erection. The penis will not get erect if the body does not provide enough NO. A penile erection results from relaxation of trabecular smooth muscle in the cavernous body and is associated with blood vessels [5] regulated by the complex interaction between local mediators and nerves. The quality of cavernosal smooth muscle and penile vascular function is controlled by vasodilators such as NO, vasoconstrictors such as noradrenaline and endothelins, and by vasoactive intestinal peptides. A molecular pathology of erectile dysfunction has been proposed, linking the function of the corpus cavernosum to the structure of the trabeculae, specifically as the ratio of connective tissue and smooth muscle trabecular must be kept for successful veno-occlusion in penile erection [6]. In a rabbit model, the alterations in this proportion were associated with atherosclerotic and hypercholesterolemic severity [7]. Many studies show that high glucose concentrations induce endothelial dysfunction in diabetes [8], and endothelial dysfunction is associated with oxidative stress [9]. Generating the hyperglycemia-dependent endothelial dysfunction is suggested by studies showing the acute effects of hyperglycemia being counterbalanced by antioxidants and free radicals $[10,11]$.

Phytotherapy has gained popularity due to more effective treatments of health problems and safe use as compared to marketed drugs [12]. Plants possessing antidiabetic activities are of significant interest in ethnobotany as they are recognized to contain valuable medicinal 
properties and a number of these plants has shown varying degrees of hypoglycemic and antihyperglycemic activities [13]. The chemical structures of a phytomolecule play a critical role in its anti-diabetic activity. The bioactive constituents found in many plant species are isolated for the direct use as drugs, lead compounds, or pharmacological agents. These traditional approaches might offer a natural key to unlock diabetic complications [14].

For several decades, curcumin, a polyphenolic natural product obtained from turmeric or diferuloylmethane molecule curcumin, has been widely used as a food coloring ingredient for its yellowish color. In the last 10 years, several in vitro and in vivo studies have reported that curcumin possesses significant pharmacological actions such as anti-tumor and antiinflammatory effects. Extensive research over the last two decades has provided evidence for the role of these different functional groups in its crucial biological activities. Curcumin exhibits therapeutic activity assigned mainly to its chemical structure and distinct biological, chemical, and physical properties against a number of diseases. Previous studies, investigated in several systems, have shown that curcumin has potent antioxidant and anti-inflammatory activities [15-18].

It is unrevealed which morphological changes the blood vessels of the penis may result from the treatment and supplementation with curcumin. In this study, a histological and morphological three-dimensional microscopic technique of vascular corrosion casting along with scanning electron microscopy (SEM) was used to study the impairment of microcirculation in the penis of induced diabetic rats. The present study aims to determine the effects of curcumin on penile blood vessels in experimentally induced diabetic rats.

\section{METHODS}

The experiment tested thirty healthy male Wistar rats with an average weight of 200-250 g. The experimental protocols and work procedure of animal research were reviewed and approved by Animal Care Committee, Medical Center, Srinakarinwirot University, Thailand. Animals were kept under standard laboratory conditions and maintained on alternate 12-hour light/dark periods $\left(25+2^{\circ} \mathrm{C}\right)$ with good lighting, moderate temperature, and adequate ventilation in a hygienic environment. They were fed standard rat chow containing proteins, carbohydrate, fats, vitamins, and minerals.

\section{Induction of diabetes}

Diabetes mellitus was experimentally induced by intraperitoneal injection of $60 \mathrm{mg} / \mathrm{kg}$ body weight of streptozotocin (Sigma, St, Louis, MO, USA) in one dose dissolved in $0.1 \mathrm{M}$ citrate buffer ( $\mathrm{pH} 4.5$ ). Diabetes was confirmed in animals 72 hours after induction by determining fasting blood glucose level $\geq 250 \mathrm{mg} / \mathrm{dl}$ using glucometer (Accu-Check ${ }^{\circ}$ Active, Roche Diagnostic, Germany) consisting of a digital meter and test strips sampling blood obtained from the tip of rat tail. Control and diabetic rats were randomly allocated to three groups: control rats (C), diabetic rats (DM), and diabetic rats supplemented with curcumin $(99.99 \%$ pure, $200 \mathrm{mg} / \mathrm{kg} \mathrm{BW}$, Sigma, St. Louis, MO, USA) (DMC) in corn oil daily. The fasting blood sugar was subsequently monitored throughout the experimental period. Animals in the control group were given only equal volume of $0.1 \mathrm{~m}$ citrate buffer used in dissolving streptozotocin intraperitoneally.

\section{Method of administration of curcumin}

The animals were fed orally using intragastric needle. The animals were held with a gloved left hand such that the neck region was held by the fingers to still the neck while being fed. The 
animals were daily treated at 7 o'clock and over a period of eight weeks they were fed 72-hour increments after induction with streptozotocin.

\section{Histological preparation for Hematoxylin and Eosin $(H \& E)$ and Masson's trichrome staining methods}

The penile tissue of the animal of all groups were fixed in Bouin's solution. They were dehydrated in graded series of ethanol through 70, 80, 90, 95 and 100\% with two changes for 1 hour each. Three changes of xylene were used as clearing reagent before filtration with 30 minutes each, embedded in paraffin, cross-sectioned at $5 \mu \mathrm{m}$ thick and stained with H\&E and Masson's trichrome staining methods. All sections were examined and photographed by an Olympus light microscope (BX-50, Olympus, Japan). The diameter and thickening of blood vessels were measured by Olympus cellSens software.

\section{Vascular Corrosion Casting Method}

The precasting procedure consisted of an intravascular injection of $0.5 \mathrm{ml}$ heparin $(5,000$ $\mathrm{IU} / \mathrm{ml}$ ) and was quickly injected into the left ventricle to prevent blood clotting. A second injection of approximately 400-500 ml normal saline solution was performed to remove all the blood out of the vascular bed. Then, Batson's no. 17 plastic mixture was immediately injected into the cannula, through the ascending aorta until the reflux from the venous vessels became evident. Each animal was left at room temperature to allow the casting medium to settle. The penises were excised and immersed in warm water $\left(80^{\circ} \mathrm{C}\right)$ to complete the hardening process. Then, the penis underwent a corrosive process in $10 \% \mathrm{KOH}$ solution at room temperature. After the tissues were cleared of, they were washed in tap water and several times with distilled water. The penile vascular cast was dissected under a stereomicroscope to obtain small specimens and let for air drying before being prepared for SEM observation (JEOL JSM-5400) at accelerating voltage of $10-15 \mathrm{KV}$.

\section{Statistical analysis}

The results were expressed as a mean + standard error of the mean. Statistical analysis was performed by using ANOVA followed by Bonferroni post-test. The value of $p<0.05$ was considered to indicate statistical significance.

\section{RESULTS}

\section{Histological Observation}

Paraffin cross-sections H\&E in control penile tissue were three erectile tissues, CS ventrally, which covered penile urethra, and the paired CC dorsally with os penis. All these structures were surrounded by a fibrous connective tissue layer, the tunica albuginea (TA). The perichondrium of the os penis was continuous with loose connective tissue of CC. There is an incomplete septum that connected with TA between the paired of CC, which allows passage of blood from one side to the other. The cavernous sinuses (venous sinus or vascular space) lined by endothelial cells were located in $\mathrm{CC}$ and the area around the os penis. The deep dorsal vein and dorsal artery of the penis were also observed (Figure 1A). 


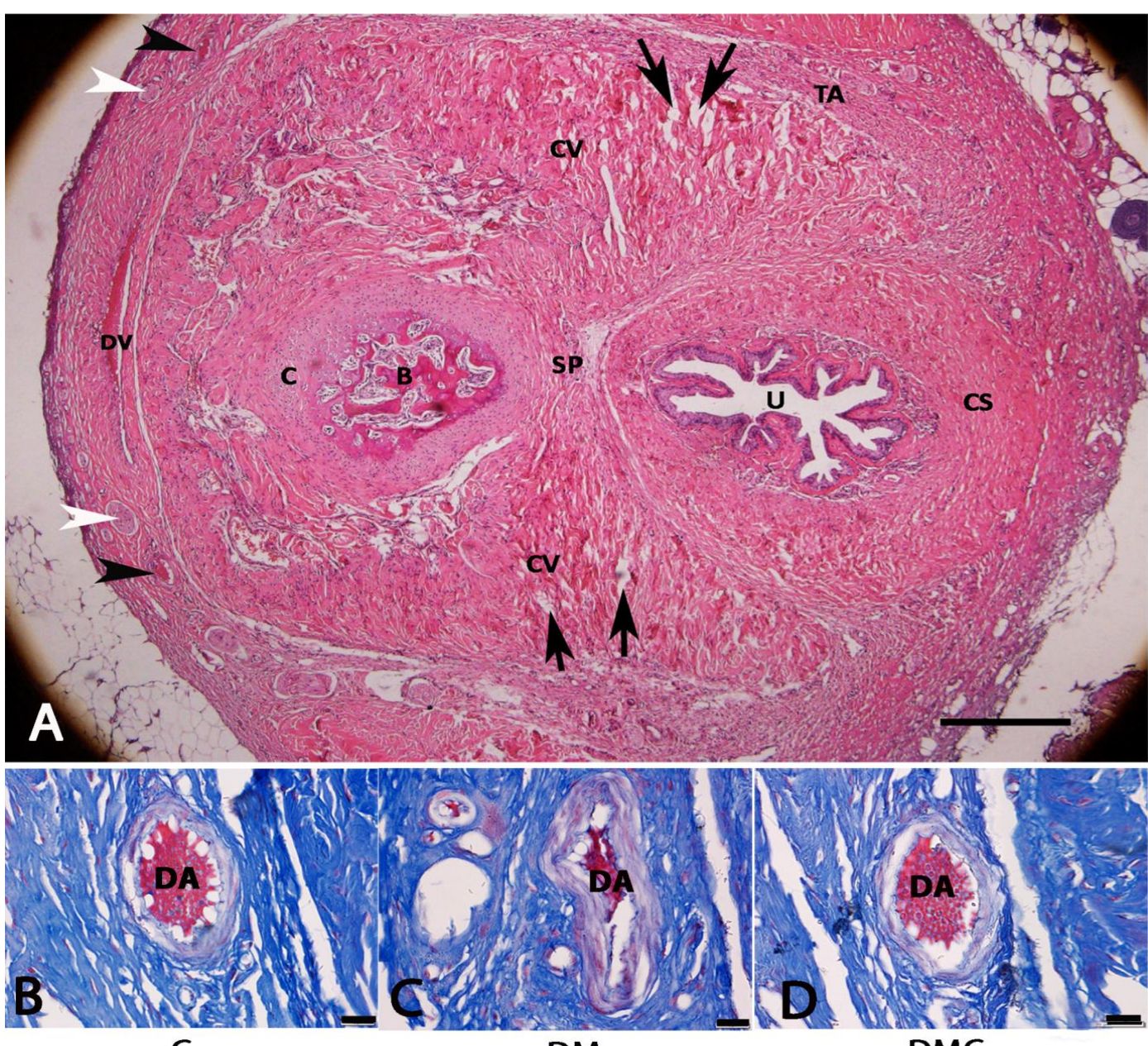

C

DM

DMC

Figure 1. Light micrographs of penile tissue of rats were stained with H\&E (A) and Masson's trichrome (B-D). (A) control group, showing cross-section of the rat penis composed of the corpus spongiosum (CS) covering penile urethra (U) and two corpus cavernosum with cavernous sinuses (arrows) bounded together in tunica albuginea (TA). Bone (B) and cartilage (C) of os penis was located anterior to septum of TA (SP) and CS. Deep dorsal vein (DV), dorsal arteries (black arrowheads) and dorsal nerve of penis (white arrowheads) were seen in the deep fascia of penis (10X). Bar $=200 \mu \mathrm{m}$. Cross-section of dorsal arteries of penis were stained with Masson's trichrome (B, C, and D). (B) control group, (C) DM group had narrow vascular lumen with thickening of collagen fibers in tunica media and (D) DMC group were also presented the increasing of diameters of dorsal $\operatorname{arteries}(60 \mathrm{X})$. Bar $=10 \mu \mathrm{m}$.

The penile tissues stained with Masson's trichrome in the control group revealed the trabeculae of the corpus cavernosum consisting of smooth muscle fibers and extracellular matrix containing mainly collagen fibers. Smooth muscle fibers were also located in the trabeculae and wall of vascular spaces, forming a subendothelial layer surrounding the venous sinus supported by a network of trabeculae (Figure 2B). In DM rats, the venous sinuses were thickly lined with layers of smooth muscle (red stain) and the muscle layers are sparse. Increasing of the thickness of collagen fibers (blue stain) was also observed. The venous sinuses were dilated and aggregated of red blood cells in the lumen (Figure 2D). The crosssection of DA was observed as narrower vascular spaces (Figure 1C) and showed thickening of collagen fibers in tunica media at the wall when compared to the dorsal arteries of the control animals (Figure 1B). Interestingly, after supplementation of curcumin in DMC rats the signs 
of vessel restoration and improvement were also presented by increased diameters and decreased collagen fibers in tunica media of DA (Figure 1D) and DV in the penile tissue. Collagen fibers in the trabeculae were decreased in thickness and they had a regular network similar to control group (Figure $2 \mathrm{~F}$ ).

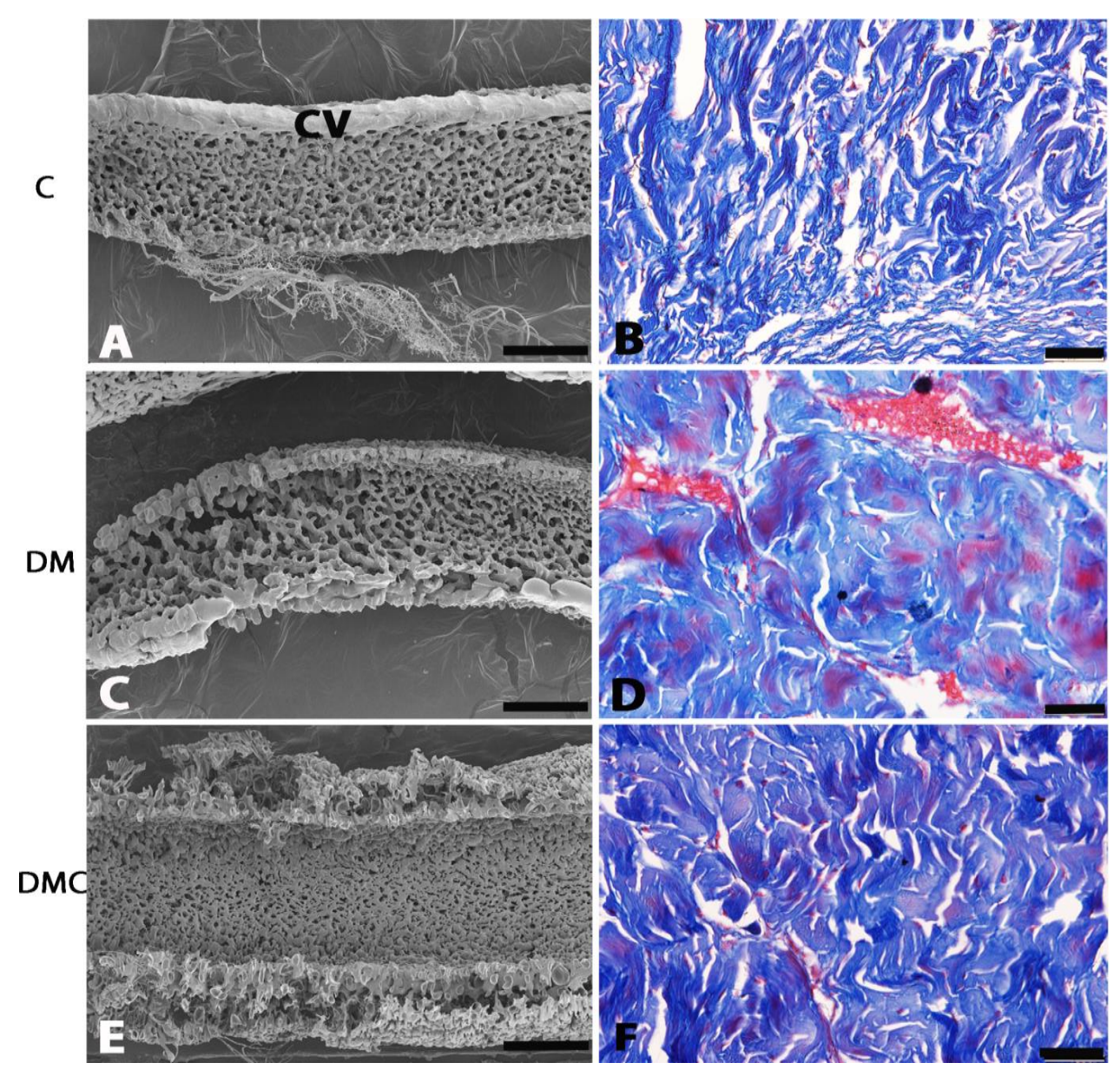

Figure 2 SEM micrographs (A, C, and E) and Light micrographs of Masson's trichrome (B, $\mathrm{D}$, and $\mathrm{F}$ ) of $\mathrm{CC}$ of rats. (A) Control group, showing vascular cast of venous sinuses in $\mathrm{CC}$ of penis. Cavernous veins $(\mathrm{CV})$ usually revealed as prominent vessels along the superior over the margins of the $\mathrm{CC}$, received communications from the cavernous spaces all along their length, (C) DM group, venous sinuses in CC were dilated and (E) DMC group, the restoration and improvement of tissues were recovering with healthy and nourishment of venous sinus Bar $=1 \mathrm{~mm}$. (B) control group revealed the trabeculae of smooth muscle fibers (red stain) and collagen fibers (blue stain). (D) DM group, the nous sinuses were thickly lined with layers of smooth muscle and the muscle layers are sparse and showed increased thickness of collagen fibers. The cavernous sinuses were dilated with aggregated red blood cells in the lumen. (F) DMC group, collagen fibers in the trabeculae were decreased in thickness and exposed regular network similar to control group (40X). Bar $=20 \mu \mathrm{m}$.

The graph data of the DM penile blood vessels showed a significant increase in diameter (Figure 6) and wall thickness of DA indices (Figure 7) when compared to control rats $(\mathrm{p}<0.001)$. In contrast, the diameters and thickness of DA were significantly decreased after treatment with curcumin in DMC rats $(\mathrm{p}<0.01, \mathrm{p}<0.001$ respectively). Additionally, the diameter (Figure 6) and thickness (Figure 7) of DV were increased; the thickness was much more severe compared to the ones of control group $(\mathrm{p}<0.01)$. Moreover, the diameter of DV were decreased after treatment with curcumin in DMC rats. 


\section{Vascular corrosion cast examination}

Vascular corrosion casting had been undertaken at low magnification of control group at 8 weeks with stereomicroscope and revealed the penis to be highly vascularized. The vascular cast of rats penis consisted of the corpus spongiosum (CS) and paired CC. CS was expanded distally to the glans penis and proximally to the markedly bifid penile bulb that covered the penile urethra. CS was supplied by a bulbourethral artery (BA). The paired crura, which merged to form the $\mathrm{CC}$, tapered distally and ended just proximally to the glans penis. In the penile shaft, anastomosed vascular spaces from each crus appeared complete (Figure 3 ). The main artery supplying the penis came from the internal pudendal artery, which branched off the three penile arteries. They were the bulbourethral, cavernosal (deep artery of the penis), and dorsal arteries of the penis. The cavernosal artery was divided into many branches, which were supported and surrounded by the trabeculae of corpora structure. The branches of the cavernosal artery closed to a capillary network and opened directly into the cavernous sinuses of the CC. In the proximal portion of the shaft, the deep dorsal veins were found along the groove of the $\mathrm{CC}$. These small vessels are closely compressed to the most superficial venous (cavernous) spaces, occasionally interconnecting them and sometimes forming closed loops near the cavernous veins (Figure 2A). Consequently, the CS, glans penis, and deep dorsal vein were separated from the $\mathrm{CC}$ as a unit during the process of corrosion with $\mathrm{KOH}$; the communications of blood vessels between the CS and CC were not apparent. The venous sinuses of the glans penis communicated with the DV at multiple sites. The rectangular cavernous spaces were oriented by communication at right angles to the deep dorsal vein (Figure 4B). The dorsal artery of the penis reached the glans penis, but careful tracing of its branches did not reveal any connection to the erectile tissues (Figure 4B and 5A).

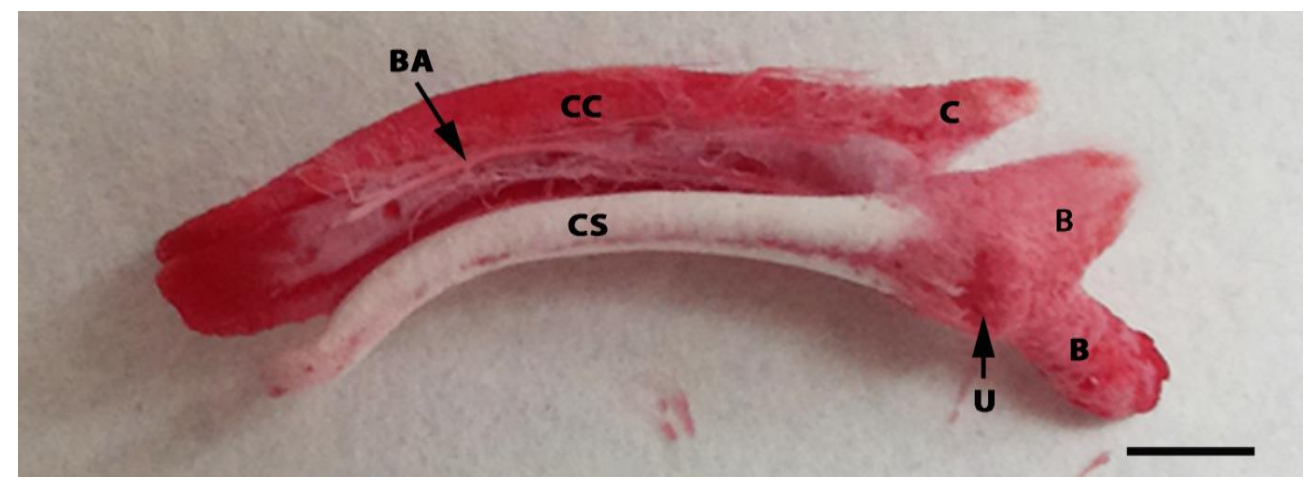

Figure 3 Photomicrograph of whole vascular cast in control rat's penis at 8 weeks. The vascular cast consisted of the CS and paired of CC. The CS was expanded distally to the glans penis (CS), glans penis, and deep dorsal vein were separated from the $\mathrm{CC}$ as a unit during the process of corrosion with $\mathrm{KOH}$ and proximally to the markedly bifid penile bulb. CS was supplied by bulbourethral artery (BA). (B) surrounded the penile urethra $(\mathrm{U})$. The paired crura $(\mathrm{C})$ merged to form the CC. Bar $=1 \mathrm{~mm}$.

The anterior branch of the cavernosal artery divided in the proximal shaft to become a small vessel in the lateral portion of the CC. The further branching of this vessel was not studied in detail. Blood supply to the shaft of the penis is provided by cavernous spaces of the crura that merge with those of the shaft and by the continuation of the anterior branch of the 
cavernosal artery. Typically, they enter the CC divided and emptied into the venous sinuses. The cavernous veins (CV), which usually revealed as prominent vessels along the superior over the margins of the $\mathrm{CC}$, received communications from the sinuses all along their length (Figure 2A). Venous drainage of the shaft of penis merged to drain into the dorsal vein of the penis.

The comparative diameter sizes of venous sinus among control, diabetic, and curcumintreated groups at 8 weeks experiment were demonstrated in the glans penis and CC (Figure 8). Diameters of DM venous sinus of glans (Figure 4C, D) were critically decreased and much more severe compared to the control group. They revealed remarkable shrinkage and lacked some parts in the distal area of the glans. The diameter of venous sinuses in CC of DM rats was dilated and the density of venous sinuses was also decreased (Figure 2C). Interestingly, the signs of vessel restoration and improvement were presented by increased diameters and healthy and nourishing of venous sinus at the area of the glans and CC of the penis in the DMC group (Figure 4E, 4F, and 2E respectively).

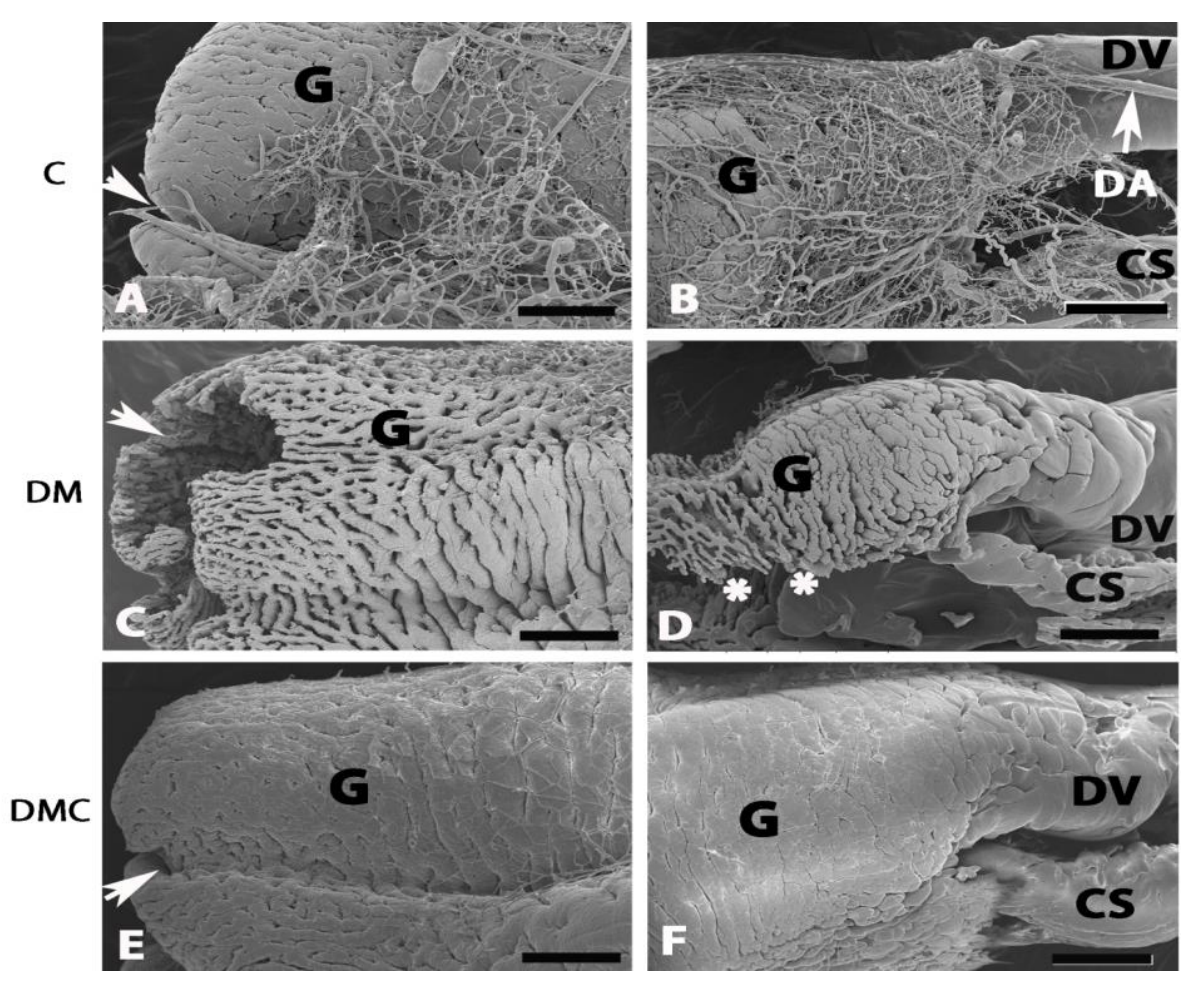

Figure 4. SEM micrographs of glans of penis $(\mathrm{G})$ vascular cast in rats at 8 weeks experiment. (A, B) Control group, illustrating venous sinus in the area of glans penis $(G)$ and drained the blood into the deep dorsal veins (DV), (B, C) DM group, destroyed venous sinuses were presented by the lack of venous sinus at distal area of the glans (white arrows). (E, F) DMC group, the redevelopment and restoration of venous sinuses were evidently. $\mathrm{CS}=$ Corpus spongiosum, white arrows = External urethral orifice, $\mathrm{Bar}=100 \mu \mathrm{m}$.

The graph data of the DM venous sinuses of glans penis showed the significantly increased diameter (Figure 8) when compared to control rats $(\mathrm{p}<0.001)$. In contrast, the diameters of venous sinuses were significantly decreased after treatment with curcumin in DMC rats $(\mathrm{p}<0.001)$. Although diameters of venous sinuses in $\mathrm{CC}$ showed no difference among the three groups of rats, the diameter was increased compared to the ones of the control group and was decreased after treatment with curcumin in DMC rats. 


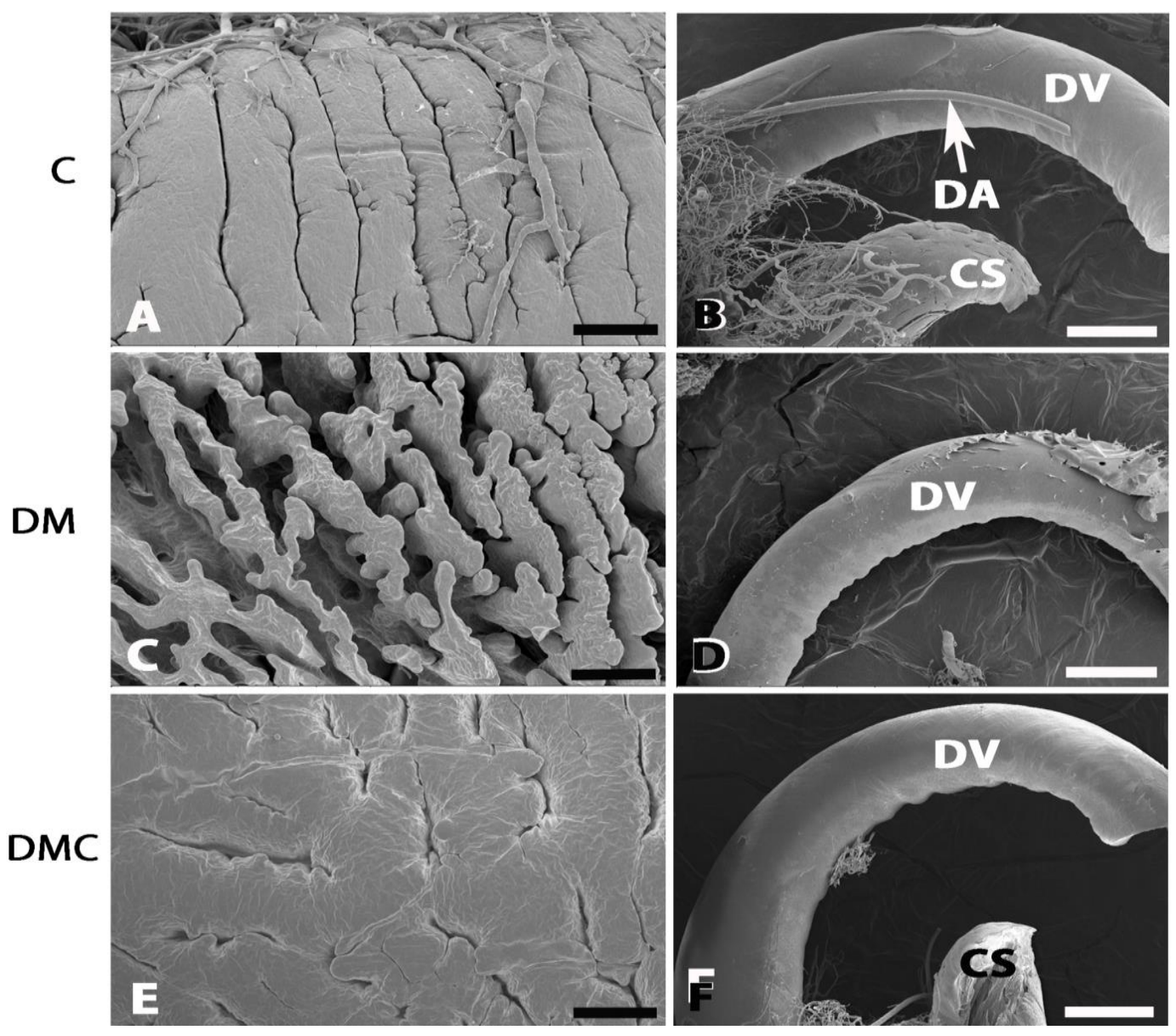

Figure 5 SEM high magnification micrographs of venous sinuses in the area of glans penis (A, C, and E) and deep dorsal veins (DV) (B, D, and F) vascular cast in rats. (A, B) Control group, (B, C) DM group, destroyed venous sinuses and small DV. (E, F) DMC group, the redevelopment and restoration of venous sinuses were evidently. $\mathrm{CS}=$ Corpus spongiosum, $\mathrm{DA}=$ Dorsal artery of penis. $(\mathrm{A}, \mathrm{C}$, and $\mathrm{E}) \mathrm{Bar}=200 \mu \mathrm{m},(\mathrm{B}, \mathrm{D}$, and F) $\mathrm{Bar}=1 \mathrm{~mm}$.
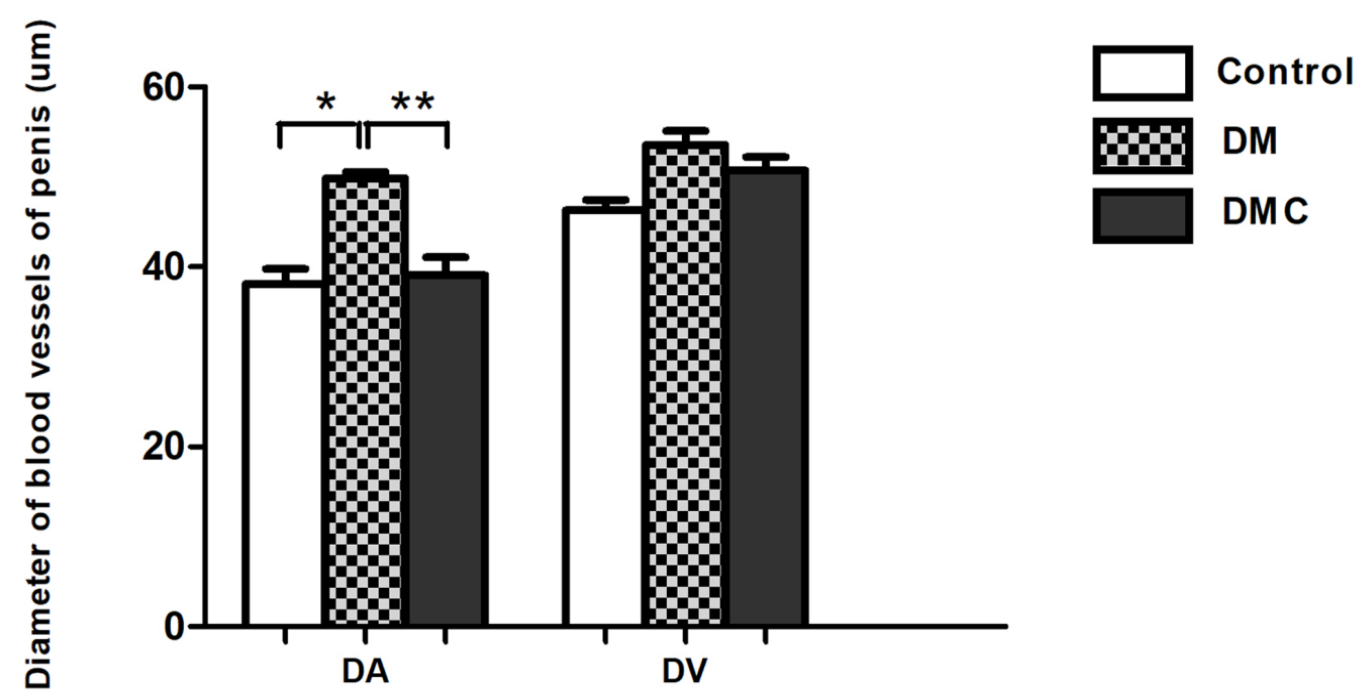

Figure 6 The average diameters of blood vessels of penis: dorsal arteries of penis (DA), deep dorsal veins $(\mathrm{DV})$ in control $(\mathrm{C})$, diabetes $(\mathrm{DM})$, and diabetes treated with curcumin (DMC) rats at 8 weeks. Values are mean $+\mathrm{SE}, * \mathrm{P}<0.001, * * \mathrm{P}<0.01$ 


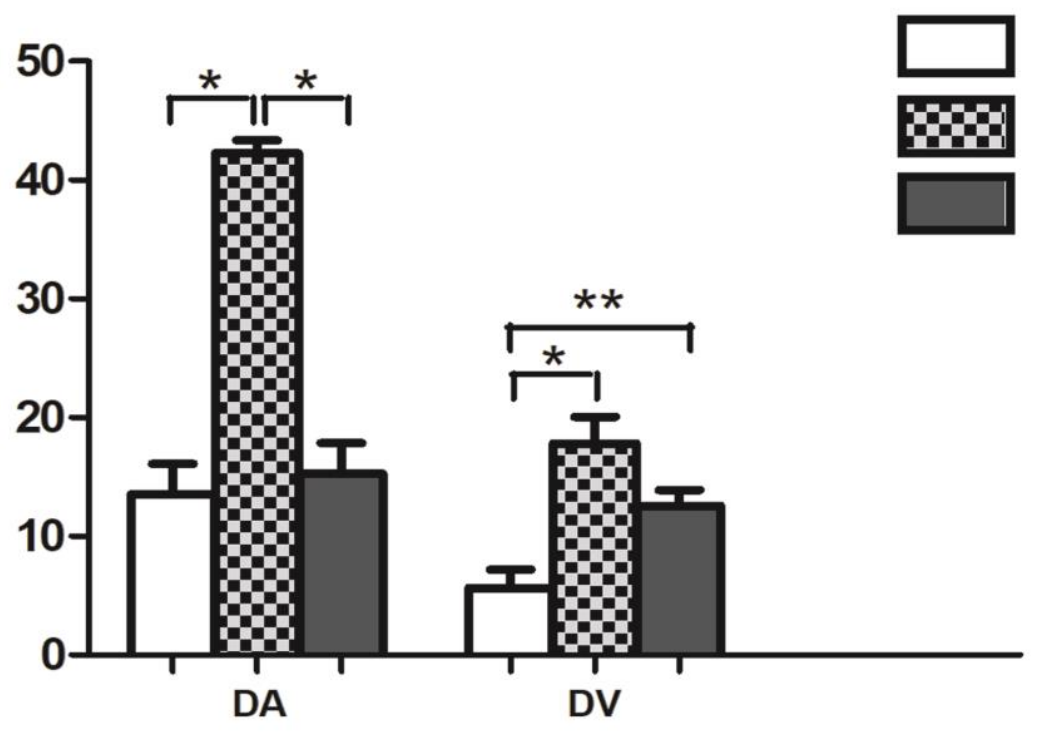

Control

DM

DMC

Figure 7 The average thickness of blood vessels of penis: dorsal arteries of penis (DA), deep dorsal veins (DV) in control (C), diabetes (DM), and diabetes treated with curcumin (DMC) rats at 8 weeks. Values are mean $+\mathrm{SE}, * \mathrm{P}<0.001, * * \mathrm{P}<0.01$

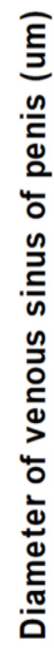
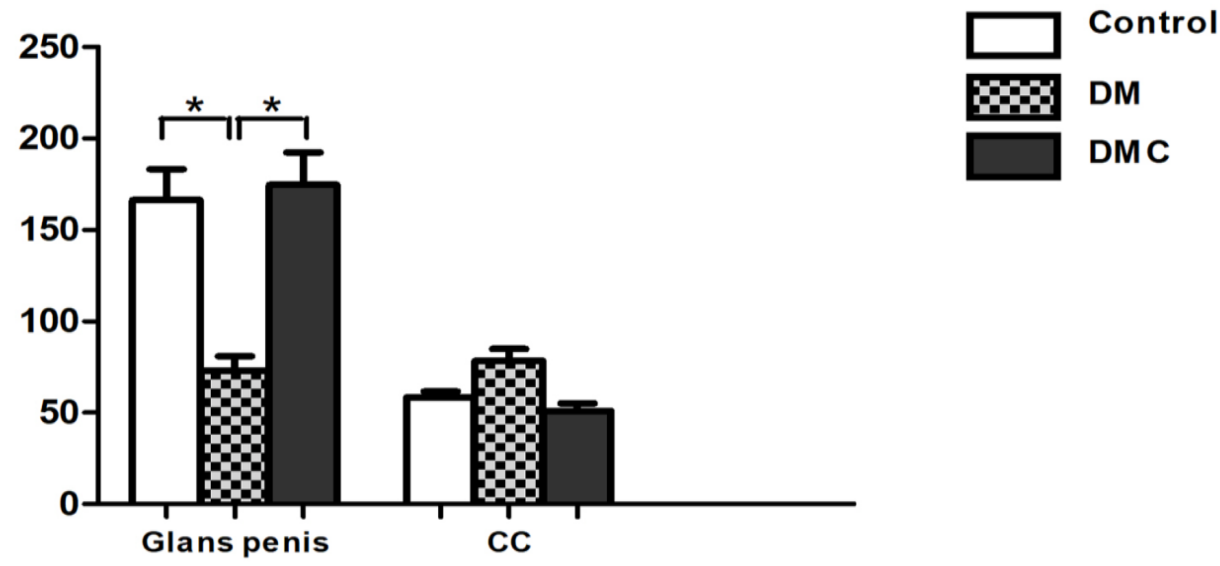

Figure 8 The average diameters of venous sinuses of penis: glans of penis, corpus cavernosum (CC) in control (C), diabetes (DM), and diabetes treated with curcumin (DMC) rats at 8 weeks. Values are mean $+\mathrm{SE}, * \mathrm{P}<0.001$

\section{DISCUSSION}

In the present study, experimental diabetes was induced by streptozotocin (STZ), led to the alteration of penile tissue in the animals by comparing the characteristics of blood vessels and tissues among three groups of rat (control, DM, and DMC). Masson's trichrome staining in DM group indicated increasing in trabecular collagen fiber content, and the smooth muscle layers were sparse in CC tissues. Degradation in corporal smooth muscle quantity and quality leads to veno-occlusive dysfunction. In Masson's trichrome sections of penile cavernosal tissue representing muscular degeneration similar to venogenic ED in human, and collagen fibers 
were the most abundant component of the trabeculae accompanied with a decrease in smooth muscle area. They appeared as wavy bundles of fibers [19].

Increasing of collagen synthesis associated with penile fibrosis was caused by hypoxia because of overexpression of tumor growth factor- $\beta 1$ (a pleiotrophic cytokine) [20]. TGF- $\beta$ is a key mediator of fibrosis [21]. The increase of extracellular matrix (ECM) protein production results in myofibroblast-made alteration of synthetic phenotype producing ECM of the original cell components in the penis. The appearance and aggregation of myofibroblasts in the penis is caused from the deposition of ECM and collagen fibers that characterize tissue fibrosis, associated with many conditions, such as in diabetes, smoking and aging.

This may explain the fibroblasts' infiltration observed in the venogenic ED sections [22]. In the study of ED in type II diabetic db/db mice it was stated that veno-occlusive dysfunction might also result from the significant alteration in collagen and elastin; both types are necessary for penile dispensability and the maintained rigidity characteristic of erection in cavernosum of $\mathrm{db} / \mathrm{db}$ mice [23].

The partial pressure of oxygen or oxygen tension played an important role in the control of the construction of cavernosal smooth muscles and collagen fibers [24]. This might explain the increased collagen and the decreased elastic fiber content observed in the venogenic ED group. I-NOS influenced the erectile tissue under hypoxic conditions. Studies of other organs showed that i-NOS was induced in response to a variety of cytokines, including hypoxiainducible factor $1 \mathrm{a}$, interleukin 1-B, interleukin-6 and tumor necrosis factor a [25, 26].

The dorsal arteries presented a dilatation of diameter, narrower vascular spaces and had thickening of collagen fibers in tunica media of the wall of them. The venous sinuses in CC were dilated and displayed with Masson's trichrome staining and vascular casting methods with SEM. The loss of cellular function is caused by the excessive deposition of extracellular matrix, especially collagen fibers deposition accompanied by the characteristic tissue fibrosis as the harmful state of overload deposition of fibrous connective tissue. The core of an excessive wound healing response frustrated the normal organ function.

$\mathrm{CS}$, erectile tissue located around the penile urethra, seems lacking smooth muscles then leading to unlimited blood flow in the CS of the penis. In contrast, CC was the main erectile structure used during erection [27]. The venous sinuses network of the penile erectile tissue was a complex network of vascular spaces. Without appropriate corporeal volume expansion, the veno-occlusive mechanism may be impaired as a result of insufficient engorgement of the corpora to occlude draining sinusoidal veins against the tunica. Complete erection of the penis might be dependent on the participation of the bulbospongiosus muscle extending the penile bulb and the ischiocavernosus muscle which encloses the crura. The cause of sexual dysfunction in diabetic patients is still in controversial, although erectile dysfunction might be due to penile nerve or blood vessel damage. In men with long-term diabetes, proliferation processes of fibrous tissue could occur in the decreased vascular volume. In non-diabetic patients, erection can be impaired as a result of atherosclerotic vascular changes [28]. The atrophy and degeneration of erectile smooth muscle cells presented with electron microscopy in diabetic rats could similarly occur in diabetic men.

By vascular cast technique, microvasculature of diabetic rat's penis undergoes severe pathological changes. Diameters of DM venous sinus vascular cast of glans penis were critically decreased and revealed remarkable shrinkage and lacked some parts in the distal area so that the density of venous sinuses was also decreased in the glans. Vascular destruction associated with diabetic incompetence caused limited blood flow into the peripheral venous 
sinuses in the glans penis [29]. It was presumed that reduction in vessel diameters could decrease the arterial blood flow induced in tissue ischemia. Furthermore, changes in the trabecular structure may also impair the veno-occlusive mechanism, predisposing to diffuse venous leakage [30, 31].

High concentrations of vasoactive intestinal peptide (VIP) in the nervous control of male external genitalia were found in autonomic nerves around the pudendal arteries and erectile tissues of the corpus cavernosum. Trabecular smooth muscle relaxation, arterial dilatation, and venous compression were three events of an erection following sexual arousal and releasing of nitric oxide to the erectile tissue [32]. Blood in the venous spaces compressed subtunical venules during erection, thereby reducing venous flow. With normal erectile function, a high intracavernous pressure was maintained with a low inflow rate [33]. The result is a flaccid state and an increased propensity for venous leakage. However, an important alternative and likely complementing factor is the ability of the trabecular smooth muscle network to relax in response to vasodilating agents, which is also impaired by diabetic disease and thus functionally decreases vascular volume and venous sinus filling. Other researchers allocated a role in collagen accumulation and apoptosis of erectile tissue as the increase of i-NOS expression was accompanied by aging in rat erectile tissue [34].

Hyperglycemia affects endothelial functions by the synthesis of growth factors and vasoactive agents in diabetes [35]. High blood sugar activates protein kinase-C (PKC) that induces phosphorylation of p115RhoGEF, a guanine nucleotide exchange factor for Rho GTPase [36]. Active RhoA is implicated in arginase induction leading to decrease in nitric oxide (NO) bioavailability [29-31]. Furthermore, activated PKC leads to sustained increases in the production of superoxide anion (O2•) that activates NF-K $\beta$ and affecting the expression of endothelial NOS (eNOS) endothelin-1 (ET-1), vascular endothelial growth factor, transforming growth factor-b and plasminogen activator inhibitor-1 [37, 38].

The effect of curcumin from turmeric on the penile tissue in STZ-induced diabetes was investigated. In an animal study, curcumin, or active compounds from turmeric, significantly improved the sensitivity of erectile function. It worked by stimulating enzymes and natural antioxidant activity which directly affected penile tissue. Curcumin administration also could improve diabetes-induced endothelial dysfunction via a decrease in vascular superoxide production and $\mathrm{PKC}$ inhibition. Curcumin induces de novo synthesis of phase II-detoxifying genes including HO-1 gene. A water-soluble derivative of curcumin could mediate erectile function via induction of HO-1 enzyme with upregulating cavernous tissue cGMP levels. A previous clinical study showed that curcumin application significantly reduced the levels of malondialdehyde, endothelin (ET-1), interleukin-6 (IL-6), and TNF- $\alpha$ in type 2 diabetes patients [39]. Other investigators reported that $\mathrm{HO}$ preserved NO through scavenging of reactive oxygen species (ROS), preventing the formation of peroxynitrite and subsequent degradation of NO. It has been reported that erectile response was a condition on NO generated by NO synthase (NOS) enzyme of the nerves and vascular endothelium in the cavernosal tissue. The nitric oxide/cyclic guanosine monophosphate (NO/cGMP) pathway and $\mathrm{H}_{2} \mathrm{~S}$ were pathways in mediating relaxation of corpus cavernosum smooth muscle, significantly relaxing vascular and intestinal preparations in vitro [40, 41]. Overexpression of HO-1 might mediate a decrease in iNOS and increase in eNOS with improvement of vascular responses in diabetic rats [42]. Curcumin as an inducer of $\mathrm{HO}-1$ could indirectly potentiate eNOS effects on vascular endothelium [43]. This work demonstrated the possible effects of curcumin as a restorative agent on penile diabetic problems. Considering the administration of curcumin in the daily diet could be recommended for its antioxidant and anti-inflammatory properties [44]. 


\section{CONCLUSION}

Our results have shown that the effects of curcumin could improve the destruction of the penile tissues and blood vessels in STZ-induced diabetic rats. This study could be valuable in considering the role of curcumin as a natural treatment for the damage of penis and vascular tissue caused by diabetes.

List of Abbreviations: C, control; DM, diabetes; STZ, streptozotocin; SEM, scanning electron microscope; CC, corpus cavernosum; CS, corpus spongiosum; DA, dorsal arteries of penis; DV, deep dorsal veins; CV, cavernous veins.

Competing Interests: All authors have no competing interests to report

Authors' Contributions: VA and WK designed and conducted the research. PB and MK assisted for tissue preparation and staining methods. WK conducted the vascular corrosion cast technique and performed statistical analysis. VA and WK wrote the manuscript. All authors read and approved the final version of the manuscript.

Acknowledgments and Funding: The authors would like to thank Mrs. Anna Chatthong, Faculty of Science, Prince of Songkla University, Hat Yai, Songkhla, Thailand for assistance with the English.

\section{REFERENCES}

1. Vinik AI, Erbas T: Recognizing and treating diabetic autonomic neuropathy. Cleve Clin J Med 2001, 68(11): 928-944.

2. Cameron NE, Cotter MA: Erectile dysfunction and diabetes mellitus: mechanistic considerations from studies in experimental models. Curr Diab Rev 2007, 3(3): 149158.

3. Nacci C, Tarquinio M, De Benedictis L, et al.: Endothelial dysfunction in mice with streptozotocin-induced type 1 diabetes is opposed by compensatory overexpression of cyclooxygenase-2 in the vasculature. Endocrinology 2009, 150(2): 849-61.

4. Angulo J, Gonzalez-Corrochano R, Cuevas P, et al.: Diabetes exacerbates the functional deficiency of NO/cGMP pathway associated with erectile dysfunction in human corpus cavernosum and penile arteries. Sex Med 2010, 7: 758-68.

5. Gratzke C, Angulo J, Chitaley K, et al.: Anatomy, physiology, and pathophysiology of erectile dysfunction. J Sex Med 2010, 7: 445-475.

6. Nehra A, Barrett DM, Moreland RB: Pharmacotherapeutic advances in the treatment of erectile dysfunction. Mayo Clin Proc 1999, 74(7): 709.

7. Nehra A, Azadzoi KM, Moreland, RB, et al.: Cavernosal expandability is an erectile tissue mechanical property which predicts trabecular histology in an animal model of vasculogenic erectile dysfunction. J Urol 1998, 159(6): 2229.

8. Adhikari S, Indira PK, Mukherjee T: Physico-chemical studies on the evaluation of the antioxidant activity of herbal extracts and active principles of some Indian medicinal plants. J Clin Biochem Nutr 2007, 40(3): 174-183. 
9. Heitzer T, Schlinzig T, Krohn K, et al.: Endothelial dysfunction, oxidative stress, and risk of cardiovascular events in patients with coronary artery disease. Circulation 2001, 104(22): 2673-2678.

10. Marfella R, Verrazzo G, Acampora R, et al.: Glutathione reverses systemic hemodynamic changes by acute hyperglycemia in healthy subjects. Am J Physiol 1995, 268: E1167-E1173.

11. Ting HH, Timimi FK, Boles KS, Creager SJ, Ganz P, Creager MA: Vitamin C improves endothelium-dependent vasodilation in patients with non-insulin dependent diabetes mellitus. J Clin Invest 1996, 97(1):22-28.

12. Ceriello A: New insights on oxidative stress and diabetic complications may lead to a "causal" antioxidant therapy. Diabetes Care 2003, 26(5): 1589-1596.

13. JK Grover, Yadav S, Vats V: Medicinal plants of India with anti-diabetic potential, Journal of Ethnopharmacology 2002, 81 (1): 81-100.

14. Babu PA, Suneetha, G: A database of 389 medicinal plants for diabetes. Bioinformation 2006, 1(4): 130-131.

15. Xu YX, Pindolia KR, Janakiraman N, Noth CJ, Chapman RA, Gautam SC: Curcumin, a compound with anti-inflammatory and anti-oxidant properties, downregulates chemokine expression in bone marrow stromal cells. Exp Hematol 1997, 25(5): 413-422.

16. Reddy AC, Lokesh BR: Studies on anti-inflammatory activity of spice principles and dietary n-3 polyunsaturated fatty acids on carrageenan-induced inflammation in rats. Ann Nutr Metab 1994, 38(6): 349-58.

17. Ammon HP, Safayhi H, Mack T, Sabieraj J: Mechanism of anti-inflammatory actions of curcumine and boswellic acids. J Ethnopharmacol 1997, 38(2-3): 113-9.

18. Joe B, Lokesh BR: Effect of curcumin and capsaicin on arachidonic acid metabolism and lysosomal enzyme secretion by rat peritoneal macrophages. Lipids 1997, 1 32(11): 1173-80.

19. Radwana DM, Kamelb IE: A histological and immunohistochemical study on human penile cavernosal tissue with viewpoints of venogenic erectile dysfunction. The Egyptian Journal of Histology 2011, 34: 92-102.

20. Moreland RB: Is there a role of hypoxemia in penile fibrosis: a viewpoint presented to the Society for the Study of Impotence. Int J Impot Res 1998, 10(2): 113-120.

21. Burke JP, Jacobson DJ, McGree ME, et al.: Diabetes and sexual dysfunction: results from the Olmsted County study of urinary symptoms and health status among men. J Urol 2007, 177(4): 1438-42.

22. Gonzalez Cadavid NF: Mechanisms of penile fibrosis. J Sex Med 2009, 6 (Suppl 3): 353-362.

23. Drury RAB, Wallington EA: Orcein stain for elastic fibres. In Carleton's histological technique. 4th edition. Edited by Drury RAB, Wallington EA. London: Oxford University Press; 1976: 177-178

24. Moreland RB: Pathophysiology of erectile dysfunction: the contributions of trabecular structure to function and the role of functional antagonism. Int J Impot Res 2000, 12 (Suppl 4): S39-S46.

25. Azadzoi KM, Master TA, Siroky MB: Effect of chronic ischemia on constitutive and inducible nitric oxide synthase expression in erectile tissue. J Androl 2004, 25(3): 382-388. 
26. Bancroft JD, Cook HC: Immunocytochemistry. In Manual of histological techniques and their diagnostic application. 2nd edition. Edited by Bancroft JD, Cook HC, Turner DR. London: Churchill Livingstone 1994: 263-325.

27. Gumus B, Vatansever HS, Muezzinoglu T, Muftuoglu S, Kaymaz F, Buyuksu C: Histopathological effects of sildenafil citrate on rat corpus cavernosum. Acta Histochem 2004, 106(1):37-45.

28. Ignarro LJ, Bush PA, Buga GM, Wood KS, Fukuto JM, Rajfer J: Nitric oxide and cyclic GMP formation upon electrical field stimulation cause relaxation of corpus cavernosum smooth muscle. Biochem Biophys Res Commun 1990, 170(2): 843850.

29. Kim SC, Ahn SY, Park SH, et al.: A comparison of the relaxation responses of isolated cavernosal smooth muscles by endothelium-independent and endotheliumdependent vasodilators in diabetic men with impotence. J Korean Med Sci 1995, 10(1): 1 .

30. Nehra A, Goldstein I, Pabby A, et al.: Mechanisms of venous leak in erectile dysfunction in patients with vascular risks factors: a clinicopathologic correlation of corporal venoocclusion. J Urol 1996, 156: 1320.

31. Nehra A, Azadzoi KM, Moreland RB, et al.: Cavernosal expandability is an erectile tissue mechanical property which predicts trabecular histology in an animal model of vasculogenic erectile dysfunction. J Urol 1998, 159(6): 2229.

32. Kim N, Azadzoi KM, Goldstein I, Saenz de Tejada I: A nitric oxide-like factor mediates nonadrenergic-noncholinergic neurogenic relaxation of penile corpus cavernosum smooth muscle. J Clin Invest 1991, 88(1): 112-118.

33. Karadeniz T, Ariman A, Topsakal M, Eksioglu A, Engin T, Basak D: Value of color Doppler sonography in the diagnosis of venous impotence. Urol Int 1995, 55(3): 143-146.

34. Ferrini M, Magee TR, Vernet D, Rajfer J, Gonzalez Cadavid NF: Aging-related expression of inducible nitric oxide synthase and markers of tissue damage in the rat penis. Biol Reprod 2001, 64(3): 974-982.

35. Yang G, Lucas R, Caldwell R, Yao L, Romero MJ, Caldwell RW: Novel mechanisms of endothelial dysfunction in diabetes. J Cardiovasc Dis Res 2010, 1(2): 59-63.

36. Xu J, Zou MH: Molecular insights and therapeutic targets for diabetic endothelial dysfunction. Circulation 2009, 120(13): 1266-1286.

37. Shamloul R: The potential role of the heme oxygenase/carbon monoxide system in male sexual dysfunctions. J Sex Med 2009, 6(2): 324-333.

38. Ryter SW, Otterbein LE, Morse D, Choi AM: Heme oxygenase/carbon monoxide signaling pathways: Regulation and functional significance. Mol Cell Biochem 2002, 234- 235: 249-63.

39. Usharani P, Mateen A, Naidu M, Raju Y, Chandra N: Effect of NCB-02, Atorvastatin and placebo on endothelial function, oxidative stress and inflammatory markers in patients with T2 diabetes mellitus: A randomized, parallelgroup, placebocontrolled, 8-week study. Drug in R and D 2008, 9(4): 243-50.

40. Burnett AL: Novel nitric oxide signaling mechanisms regulate the erectile response. Int J Impot Res 2004, 16: S15-S19. 
41. Teague B, Asiedu S, Moore PK: The smooth muscle relaxant effect of hydrogen sulphide in vitro: evidence for a physiological role to control intestinal contractility. Br J Pharmacol 2002, 137(2): 139-45.

42. Ahmad M, Turkseven S, Mingone CJ, Gupte SA, Wolin MS, Abraham NG: Heme oxygenase-1 gene expression increases vascular relaxation and decreases inducible nitric oxide synthase in diabetic rats. Cell Mol Biol 2015, 51(4): 371-376.

43. Shamloul R: The potential role of the heme oxygenase/carbon monoxide system in male sexual dysfunctions. J Sex Med 2009, 6(2): 324-333.

44. Chuengsamarn S, Rattanamongkolgul S, Luechapudiporn R, Phisalaphong C, Jirawatnotai S: Curcumin extract for prevention of type 2 diabetes. Diabetes Care 2012, 35(11): 2121-7. 